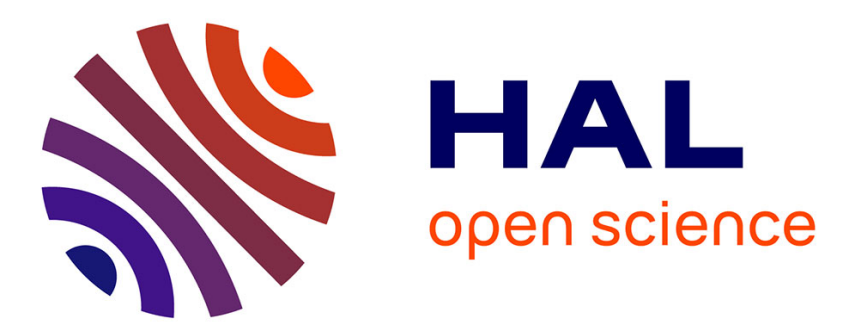

\title{
Conjugate gradient Mojette reconstruction
}

\author{
Myriam Servières, Jérôme Idier, Nicolas Normand, Jean-Pierre Guédon
}

\section{To cite this version:}

Myriam Servières, Jérôme Idier, Nicolas Normand, Jean-Pierre Guédon. Conjugate gradient Mojette reconstruction. SPIE Medical Imaging 2005: Image Processing, Feb 2005, San Diego, CA, United States. pp.2067-2074, 10.1117/12.593399 . hal-01500580

\section{HAL Id: hal-01500580 \\ https://hal.science/hal-01500580}

Submitted on 16 May 2017

HAL is a multi-disciplinary open access archive for the deposit and dissemination of scientific research documents, whether they are published or not. The documents may come from teaching and research institutions in France or abroad, or from public or private research centers.
L'archive ouverte pluridisciplinaire HAL, est destinée au dépôt et à la diffusion de documents scientifiques de niveau recherche, publiés ou non, émanant des établissements d'enseignement et de recherche français ou étrangers, des laboratoires publics ou privés. 


\title{
Conjugate Gradient Mojette Reconstruction
}

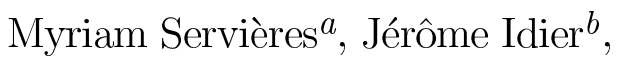 \\ Nicolas Normand ${ }^{a}$ and JeanPierre Guédon ${ }^{a}$ \\ ${ }^{a}$ IRCCyN/IVC - UMR CNRS 6597, Image \& Video Communication, \\ École polytechnique de l'Université de Nantes, \\ La Chantrerie, BP 50609, 44306 Nantes Cedex 3 \\ ${ }^{b} \mathrm{IRCCyN} / \mathrm{ADTS}$, \\ École Centrale de Nantes \\ 1 rue de la Noë, 44321 NANTES Cedex 3
}

\begin{abstract}
Iterative methods are now recognized as powerful tools to solve inverse problems such as tomographic reconstruction. In this paper, the main goal is to present a new reconstruction algorithm made from two components. An iterative algorithm, namely the Conjugate Gradient (CG) method, is used to solve the tomographic problem in the least square (LS) sense for our specific discrete Mojette geometry. The results are compared (with the same geometry) to the corresponding Mojette Filtered Back Projection (FBP) method.

In the fist part of the paper, we recall the discrete geometry used to define the projection $M$ and backprojection $M^{*}$ operators. In the second part, the CG algorithm is presented within the context of the Mojette geometry. Noise is then added onto these Mojette projections with respect to the sampling and reconstructions are performed. Finally the Toeplitz block Toeplitz (TBT) character of $M^{*} M$ is demonstrated.
\end{abstract}

\section{INTRODUCTION}

Medical tomographic devices are mostly driven by the popular FBP algorithm. Iterative methods have proven their ability to properly invert the projection data by incorporating a priori properties as well as the geometry of the acquisition or the characteristics of the task that the physician has to perform on the final image. For instance, during the last decade, J. Fessler and his colleagues have gave some understanding of iterative scheme ${ }^{3}$ applied to the minimization of penalized least square criteria. Our prime goal in this paper is to start over with a new discrete geometry that does not correspond to the way data are collected today but rather gives the discrete problem a simpler mathematical expression. This expression has been tested for direct inversion using the zero degree spline FBP algorithm ${ }^{4}$ in another paper of these proceedings. ${ }^{7}$ Conversely, the use of a simple but iterative algorithm using the same discrete geometry modeling is the center of this paper. The discrete Mojette sampling geometry is recalled in section 2 where the fact that the dual operator $M^{*}$ of the projector $M$ is exact is enhanced. The adopted iterative minimization scheme is the conjugate gradient (CG) method. In section 3, the structure of the normal matrix $M^{*} M$ is studied. Finally, section 4 implements the conjugate gradient algorithm and compares noisy phantom reconstructions both for the CG and FBP algorithms. Section 5 discusses and summarizes the existing differences between direct and iterative algorithms.

\footnotetext{
Further author information: (Send correspondence to Myriam Servières)

a Email: \{myriam.servieres, nicolas.normand, jean-pierre.guedon \}@polytech.univ-nantes.fr

Phone: + 33240683 048, Fax: + 33240683232

$b$ Email: Jerome.Idier@irccyn.ec-nantes.fr

Phone: +33240 376 909, Fax: +33240376930
} 


\section{MOJETTE SAMPLING GEOMETRY}

The Mojette projection is a linear discrete exact version of the Radon transform. The projection directions are defined by discrete angles $(p, q)$ with $\tan \theta=q / p$ with $p$ and $q$ prime relatively and for $q>0 q=0, p=1$. The sampling on the projections is $t=\Delta b / \sqrt{p^{2}+q^{2}}$ where $\Delta$ is the pixel size and $b$ the bin index onto the projection. It allows for an exact backprojection with no interpolation scheme added onto the projections. ${ }^{6}$ The sampling geometry is adapted to the discrete geometry of the digital reconstructed image. The Mojette projector can be defined for several pixel models. The simpliest is a Dirac pixel model as defined as:

$$
\operatorname{proj}_{\delta}(b, p, q)=\sum_{k=-\infty}^{+\infty} \sum_{l=-\infty}^{+\infty} f(k, l) \delta(b+q k-p l) d x d y .
$$

The use of this projector with the discrete angle set $S=\{(1,0),(1,1),(-2,1)\}$ is illustrated on (Fig. 1).

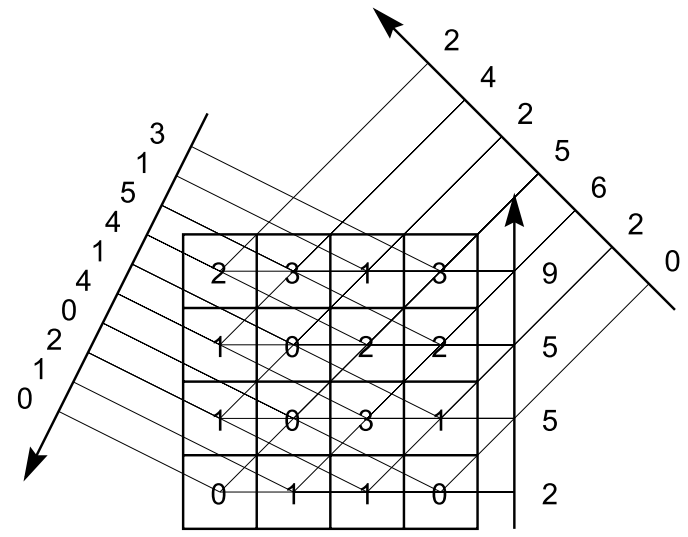

Figure 1. Mojette projections of a $4 \times 4$ image with projection directions $(p, q) \in\{(1,0),(1,1),(-2,1)\}$

Pixel $(k, l)$ of the input object contributes to the bin $b$ with a coefficient 1 when it is aligned with this bin in the $(p, q)$ direction, or 0 otherwise. Let $N \times N$ be the size of the input object. The latter can be rewritten in column vector form where the pixel index is $k+l \times N$.

The Mojette exact backprojector is defined $\mathrm{as}^{6}$ :

$$
\left[M^{*} \operatorname{proj}(b, p, q)\right](k, l)=\sum_{i=-\infty}^{+\infty} \sum_{j=-\infty}^{+\infty} \delta(k-i) \delta(l-j) \sum_{b=-\infty}^{+\infty} \operatorname{proj}(b, p, q) \Delta(b+q i-p j)
$$

$M^{*}$ is the dual Mojette operator and the definition of $M^{*}$ shows $M^{*}=M^{T}$ where the $T$ denotes the transpose matrix.

\section{3. $M^{*} M$ PROPERTIES}

The matrix $M^{*} M$ represents the key point of the iterative scheme since we are looking to minimize $\left|M^{*} M p-M^{*} b\right|$. In this section, we first derive its formula and then presents its main characteristics. 


\subsection{Expression of $M^{*} M$}

When a pixel $(k, l)$ from a $N \times N$ image is projected in the $(p, q)$ direction onto the bin $b$ on the Mojette projection, the indexes are linked by:

$$
b+q k-p l=0
$$

To get the entries of $M^{*} M$, the starting image is written as a vector of pixels according to the lexicographic order. $M$ performs the projection of this vector of pixels onto a vector of bins, while $M^{*}$ performs the backprojection of the vector of bins into the pixel vector. The index $i$ in the pixel vector corresponds to the point $\left(k_{1}, l_{1}\right)$ with:

$$
i=k_{1}+l_{1} \times N
$$

where $N$ is the width in pixels of the starting image.

In the $M^{*} M$, matrix for a $(p, q)$ projection direction, there is a coefficient sets to 1 in the $(i, j)$ index only if pixels $i$ and $j$ are projected on the same bin in the $(p, q)$ direction.

The index in the $M^{*} M$ matrix can be defined with index on the starting image.

$$
\left\{\begin{array} { l } 
{ i = k _ { 1 } + l _ { 1 } \times N } \\
{ j = k _ { 2 } + l _ { 2 } \times N }
\end{array} \text { with } \left\{\begin{array}{l}
l_{1}=\left\lfloor\frac{i}{N}\right\rfloor \\
l_{2}=\left\lfloor\frac{j}{N}\right\rfloor
\end{array}\right.\right.
$$

where $\lfloor x\rfloor$ denotes is the integer part of $x$.

If the pixels $\left(k_{1}, l_{1}\right)$ and $\left(k_{2}, l_{2}\right)$ are projected in the same direction, this gives:

$$
\begin{aligned}
& b_{1}=-q k_{1}+p l_{1} \\
& b_{2}=-q k_{2}+p l_{2}
\end{aligned} .
$$

The index $(i, j)$ of matrix $M^{*} M$ is set to 1 if the two pixels are on the same bin if $b_{1}-b_{2}=0$, i.e. $-q\left(k_{1}-k_{2}\right)+p\left(l_{1}-l_{2}\right)=0$. That gives the formula for one projection direction of $(p, q)$ :

$$
M^{*} M_{\left(k_{1}, l_{1}\right),\left(k_{2}, l_{2}\right)}=\sum_{\#(p, q)} \Delta\left(b_{1}-b_{2}\right),
$$

where $\Delta(x)=\left\{\begin{array}{l}1 \text { if } x=0 \\ 0 \text { elsewhere. }\end{array}\right.$ (Eq. 7) can also be written with $(i, j)$ index as a sum on all the projection directions:

$$
M^{*} M_{(i, j)}=\sum_{\#(p, q)} \Delta\left[-q(i-j)+(q N+p)\left(\left\lfloor\frac{i}{N}\right\rfloor-\left\lfloor\frac{j}{N}\right\rfloor\right)\right] .
$$

With such an expression we can easily write the $M^{*} M$ matrix (Fig. 2)

\begin{tabular}{lll|lll|lll}
1 & 0 & 0 & 0 & 0 & 0 & 0 & 1 & 0 \\
0 & 1 & 0 & 0 & 0 & 0 & 0 & 0 & 1 \\
0 & 0 & 1 & 0 & 0 & 0 & 0 & 0 & 0 \\
\hline 0 & 0 & 0 & 1 & 0 & 0 & 0 & 0 & 0 \\
0 & 0 & 0 & 0 & 1 & 0 & 0 & 0 & 0 \\
0 & 0 & 0 & 0 & 0 & 1 & 0 & 0 & 0 \\
\hline 0 & 0 & 0 & 0 & 0 & 0 & 1 & 0 & 0 \\
1 & 0 & 0 & 0 & 0 & 0 & 0 & 1 & 0 \\
0 & 1 & 0 & 0 & 0 & 0 & 0 & 0 & 1
\end{tabular}

Figure 2. $M^{*} M$ expression for the $(1,2)$ direction for a $3 \times 3$ image. 
With the formalism described in ${ }^{1}$ the matrix can be written:

\begin{tabular}{ccc|ccc|ccc}
$\mathrm{f}(0,0)$ & $\mathrm{f}(1,0)$ & $\mathrm{f}(2,0)$ & $\mathrm{f}(0,1)$ & $\mathrm{f}(1,1)$ & $\mathrm{f}(2,1)$ & $\mathrm{f}(0,2)$ & $\mathrm{f}(1,2)$ & $\mathrm{f}(2,2)$ \\
$\mathrm{f}(-1,0)$ & & & $\mathrm{f}(-1,1)$ & & & $\mathrm{f}(-1,2)$ & & \\
$\mathrm{f}(-2,0)$ & & & $\mathrm{f}(-2,1)$ & & & $\mathrm{f}(-2,2)$ & & \\
\hline $\mathrm{f}(0,-1)$ & $\mathrm{f}(1,-1)$ & $\mathrm{f}(2,-1)$ & $\mathrm{f}(0,0)$ & $\mathrm{f}(1,0)$ & $\mathrm{f}(2,0)$ & $\mathrm{f}(0,1)$ & $\mathrm{f}(1,1)$ & $\mathrm{f}(2,1)$ \\
$\mathrm{f}(-1,-1)$ & & & $\mathrm{f}(-1,0)$ & & & $\mathrm{f}(-1,1)$ & & \\
$\mathrm{f}(-2,-1)$ & & & $\mathrm{f}(-2,0)$ & & & $\mathrm{f}(-2,1)$ & & \\
\hline $\mathrm{f}(0,-2)$ & $\mathrm{f}(1,-2)$ & $\mathrm{f}(2,-2)$ & $\mathrm{f}(0,-1)$ & $\mathrm{f}(1,-1)$ & $\mathrm{f}(2,-1)$ & $\mathrm{f}(0,0)$ & $\mathrm{f}(1,0)$ & $\mathrm{f}(2,0)$ \\
$\mathrm{f}(-1,-2)$ & & & $\mathrm{f}(-1,-1)$ & & & $\mathrm{f}(-1,0)$ & & \\
$\mathrm{f}(-2,-2)$ & & & $\mathrm{f}(-2,-1)$ & & & $\mathrm{f}(-2,0)$ & &
\end{tabular}

with

$$
f(a, b)=\sum_{p, q} \Delta[-q a+p b]
$$

\section{2. $M^{*} M$ is a TBT matrix}

In this paragraph we show that the Matrix $M^{*} M$ is a block-Toeplitz matrix with Toeplitz blocks (in short, TBT for Toeplitz-Block-Toeplitz).

For two pixels on the same line in the starting image translated of one row, their coefficient in the $M^{*} M$ matrix will be translated of $N$ row and $N$ column in $M^{*} M$. If these two aligned pixels are translated by one column in the starting image, their coefficient will be translated of one row and one column in $M^{*} M$.

If $(i, j)$ and $(i+1, j+1)$ belong to the same block, i.e. if $\left\lfloor\frac{i+1}{N}\right\rfloor=\left\lfloor\frac{i}{N}\right\rfloor$ and $\left\lfloor\frac{j+1}{N}\right\rfloor=\left\lfloor\frac{j}{N}\right\rfloor$ then

$$
\begin{array}{r}
-q\left(i+1-N\left\lfloor\frac{i+1}{N}\right\rfloor\right)+q\left(j+1-N\left\lfloor\frac{j+1}{N}\right\rfloor\right)+p\left\lfloor\frac{i+1}{N}\right\rfloor-p\left\lfloor\frac{j+1}{N}\right\rfloor= \\
-q\left(i-N\left\lfloor\frac{i}{N}\right\rfloor\right)+q\left(j-N\left\lfloor\frac{j}{N}\right\rfloor\right)+p\left\lfloor\frac{i}{N}\right\rfloor-p\left\lfloor\frac{j}{N}\right\rfloor,
\end{array}
$$

Thus $M^{*} M_{(i+1, j+1)}=M^{*} M_{(i, j)}$ and $M^{*} M$ is composed of Toeplitz blocks.

Furthermore, as

$$
\begin{array}{r}
-q\left(i+N-N\left\lfloor\frac{i+N}{N}\right\rfloor\right)+q\left(j+N-N\left\lfloor\frac{j+N}{N}\right\rfloor\right)+p\left\lfloor\frac{i+N}{N}\right\rfloor-p\left\lfloor\frac{j+N}{N}\right\rfloor= \\
-q\left(i-N\left\lfloor\frac{i}{N}\right\rfloor\right)+q\left(j-N\left\lfloor\frac{j}{N}\right\rfloor\right)+p\left\lfloor\frac{i}{N}\right\rfloor-p\left\lfloor\frac{j}{N}\right\rfloor,
\end{array}
$$

we deduce that $M^{*} M_{(i+N, j+N)}=M^{*} M_{(i, j)}$ and we can conclude that $M^{*} M$ is TBT.

\subsection{Conditioning of $M^{*} M$}

Since $M^{*} M$ is a square invertible matrix, its conditioning is given by:

$$
\operatorname{cond}\left(M^{*} M\right)=\left\|M^{*} M\right\| \cdot\left\|\left(M^{*} M\right)^{-1}\right\|
$$

This formula, when computed for the $L_{2}$ norm gives the result:

$$
C_{2}=\operatorname{cond}_{2}\left(M^{*} M\right)=\frac{\max _{i}\left|\lambda_{i}\right|}{\min _{i}\left|\lambda_{i}\right|},
$$

where $\lambda_{i}$ is an eigenvalue of $M^{*} M$.

For block Toeplitz matrices, asymptotic eigenvalue distribution can be derived as in ${ }^{1}$ using the Fourier domain. For instance, for a $16 \times 16$ image, Table 1 gives both the exact conditioning of $M^{*} M$ and its approximation with various numbers of angles. 
Table 1. Comparison of exact and approximated $M^{*} M$ conditioning

\begin{tabular}{|l|l|l|}
\hline angles & exact $C_{\infty}$ & approximated $C_{2}$ \\
\hline 32 & 18.04 & 28.96 \\
\hline 64 & 8.13 & 8.22 \\
\hline 128 & 3.83 & 4.74 \\
\hline 256 & 2.34 & 2.72 \\
\hline
\end{tabular}

\section{CONJUGATE GRADIENT RECONSTRUCTION}

\subsection{CG Algorithm}

Conjugate Gradient is a well-known algorithm to implement inverse problem solutions based on criterion minimization $^{3,}{ }^{2}$ We define our estimated solution $p$ as the image that minimizes the least square criterion:

$$
J(p)=\|b-M p\|^{2},
$$

where $p$ is the matrix of pixels and $b$ the vector of the projected bins. Equivalently, $p$ cancels the gradient of $J$, i.e.,

$$
M^{*} M p=M^{*} b .
$$

In the present case, the standard form of the Conjugate Gradient method reads ${ }^{5}$ :

$$
\left\{\begin{array} { l } 
{ p _ { 0 } \text { given } } \\
{ r _ { 0 } = M ^ { * } b - M ^ { * } M p _ { 0 } } \\
{ d _ { 0 } = r _ { 0 } }
\end{array} \left\{\begin{array}{l}
k=0,1 \ldots \\
\alpha_{k}=\frac{r_{k}^{T} r_{k}}{d_{k}^{T} M^{*} M d_{k}} \\
p_{k+1}=p_{k}+\alpha_{k} d_{k} \\
r_{k+1}=r_{k}-\alpha_{k} M^{*} M d_{k} \\
\beta_{k+1}=\frac{r_{k+1}^{T} r_{k+1}}{r_{k}^{T} r_{k}} \\
d_{k+1}=r_{k+1}+\beta_{k+1} d_{k}
\end{array}\right.\right.
$$

where $r_{k}=-\nabla J\left(p_{k}\right) / 2=M^{*} b-M^{*} M p_{k}$ and $\alpha_{k}, d_{k}$ are the current values of the stepsize and of the descent direction, respectively.

\subsection{Reconstruction without noise}

The first test image is a square $128 \times 128$ image with $17 \times 17$ centered pixels with a Dirichlet condition with the value 1 in the centered $15 \times 15$ square, $\frac{5}{8}$ in the boundaries and $\frac{7}{16}$ in the corners (fig.3a). The background value is $\frac{1}{4}$. The second one is a disk with a diameter of 21 pixels. The value of the pixels on the boundary of the disk depends on the intercepted surface between the pixel and the disk (fig.3b).

The Mojette operator is used to get analytical projections $b$ equally distributed on $[0, \pi[$. In such an ideal case, the tests images are perfectly reconstructed.

The FBP Spline 0 was also implemented as described in a companion paper in these proceedings. ${ }^{7}$

Table 2. Iteration number for a CG reconstruction without noise.

\begin{tabular}{|l|c|c|c|c|c|}
\hline Phantom & 32 projections & 64 projections & 128 projections & 256 projections & 512 projections \\
\hline \hline Square & 1366 & 82 & 31 & 22 & 14 \\
\hline Circle & 2785 & 147 & 35 & 24 & 16 \\
\hline
\end{tabular}




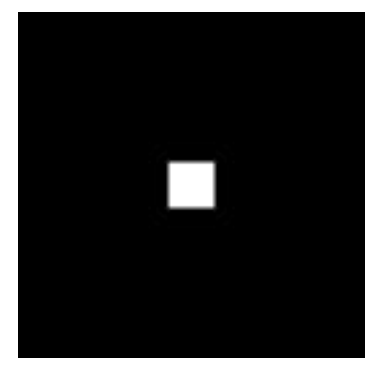

(a) square $128 \times 128$ phantom

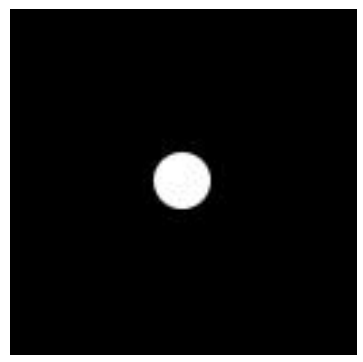

(b) disk $128 \times 128$ phantom

Figure 3. Test images

\subsection{Noisy reconstruction and comparison to FBP reconstruction}

A noise sequence following a Poisson statistics is produced and added onto the projections with respect to the number of bins per projection angle $(p, q)$.

We obtain good LS solutions on noisy projections using the CG scheme with Mojette Dirac projector and backprojector (fig. 4) onto this phantom.

Compared to FBP reconstructions (fig. 5), the LS solutions are less noisy for the same initial noise level.

The MSE is calculated onto the phantom shape on a $23 \times 23$ pixel square centered onto the square shape and on a $27 \times 27$ square centered onto the disk shape.

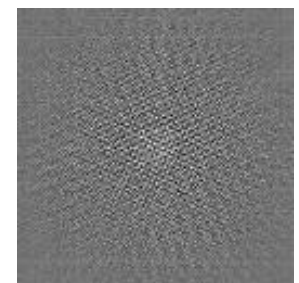

(a) Reconstructed image with Poisson noise, 32 projections, 4572 iterations, $\mathrm{MSE}=0.4259$

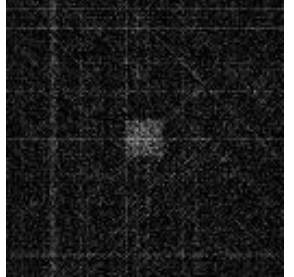

(b) Reconstructed image with Poisson noise, $\quad 64$ projections, 185 iterations, $\mathrm{MSE}=0.1766$

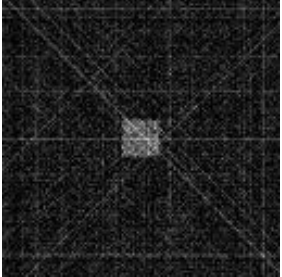

(c) Reconstructed image with Poisson noise, 128 projections, 38 iterations, $\mathrm{MSE}=0.1160$

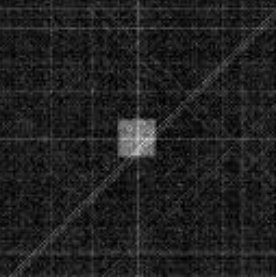

(d) Reconstructed image with Poisson noise, $\quad 256$ projections, 27 iterations, $\mathrm{MSE}=0.0900$

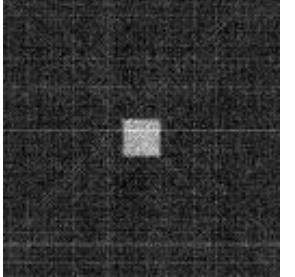

(e) Reconstructed image with Poisson noise, $\quad 512$ projections, 17 iterations, $\mathrm{MSE}=0.0446$

Figure 4. Square phantom LS solution obtained by CG with gracefully distributed discrete angles on $[0, \pi[$.

\section{DISCUSSION}

\subsection{Mojette Tomographic Geometry}

The first lesson given by using the Mojette geometry with the operators $M$ and $M^{*}$ is the stability of the results. Their behaviors both for direct and iterative methods are close to the standard sampling schemes. 


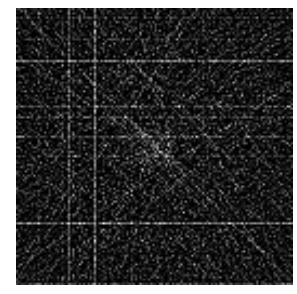

(a) Reconstructed image with Poisson noise, 32 projections, MSE $=0.2711$

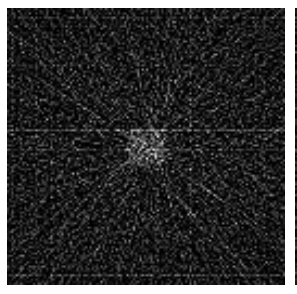

(b) Reconstructed image with Poisson noise, 64 projections, MSE $=0.2203$

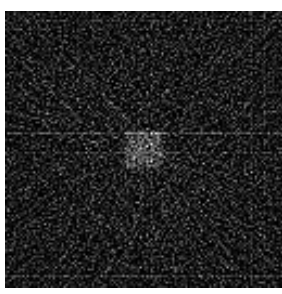

(c) Reconstructed image with Poisson noise, $\quad 128$ projections, $\mathrm{MSE}=0.2035$

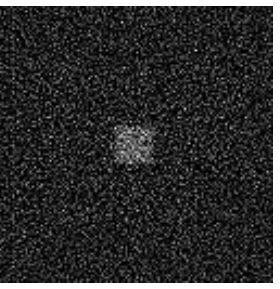

(d) Reconstructed image with Poisson noise, 256 projections, $\mathrm{MSE}=0.1867$

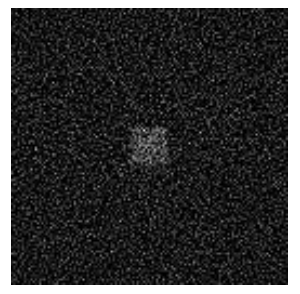

(e) Reconstructed image with Poisson noise, $\quad 512$ projections, $\mathrm{MSE}=0.2268$

Figure 5. Spline 0 square image reconstruction with uniformly distributed Katz angles on $\left[0, \pi\left[\right.\right.$ filtered by $k_{0}(b, p, q)$ and backprojected with a Mojette backprojection. The resulting images are normalized onto [0,1].

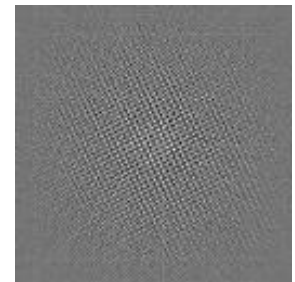

(a) Reconstructed image with Poisson noise, 32 projections, 4610 iterations, $\mathrm{MSE}=0.4259$

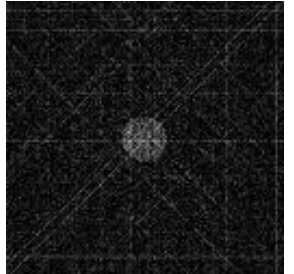

(b) Reconstructed image with Poisson noise, $\quad 64$ projections, 186 iterations, $\mathrm{MSE}=0.2813$

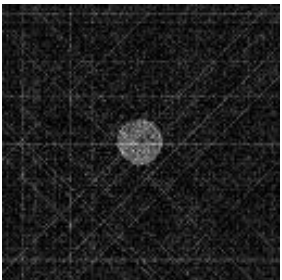

(c) Reconstructed image with Poisson noise, 128 projections, 38 iterationsMSE $=0.1799$

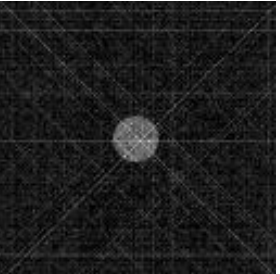

(d) Reconstructed image with Poisson noise, 256 projections, 27 iterations, $\mathrm{MSE}=0.1745$

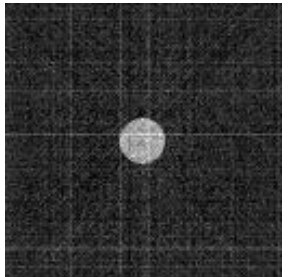

(e) Reconstructed image with Poisson noise, $\quad 512$ projections, 17 iterations, $\mathrm{MSE}=0.0625$

Figure 6. Circular phantom LS solution obtained by CG with gracefully distributed discrete angles on $[0, \pi[$.

It should be pointed out that the obtention of the initial Mojette projection sampling is not discussed in this paper. The necessary rebinning step (according to a classical 3D acquisition device) with introduce an initial supplementary smoothing. Notice that this step can also be made without interpolation as in the 3D PET acquisition.

\subsection{Noise Versus Number of Projections Behaviors}

The reconstructions and the MSE indicator computed onto the central region follow the classical results obtained with iterative LS methods and direct FBP ones. It should be stressed that for a very low number of projections, the LS approximation without penalties behaves in a very good manner but the streaks artifacts of the FBP algorithm are not much better. As soon as a correct (even low, e.g 64) number of projections is chosen (according to the size of the image), the CG-MSE will not only beat the FBP-MSE Figure of Merit but the rate of decrease is very low for the latter while the iterative CG algorithm will take benefits of the increase of conditioning of the $M^{*} M$ matrix.

Finally, the very close results between the square and disk phantoms allows for assessing the same behavior 


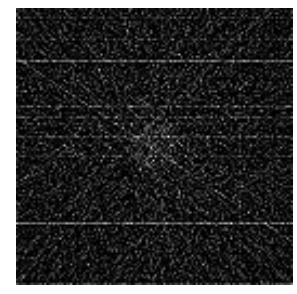

(a) Reconstructed image with Poisson noise, 32 projections, MSE $=0.4620$

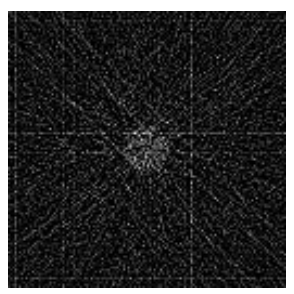

(b) Reconstructed image with Poisson noise, 64 projections, MSE $=0.4018$

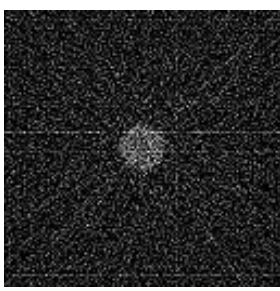

(c) Reconstructed image with Poisson noise, $\quad 128$ projections, $\mathrm{MSE}=0.3154$

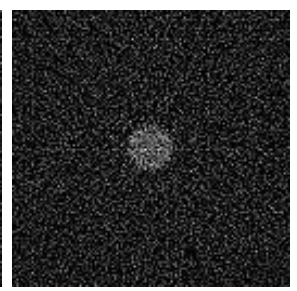

(d) Reconstructed image with Poisson noise, $\quad 256$ projections, $\mathrm{MSE}=0.3180$

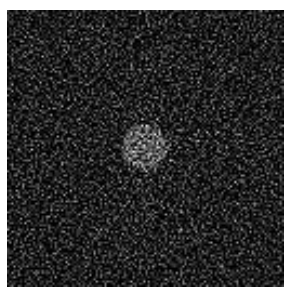

(e) Reconstructed image with Poisson noise, $\quad 512$ projections, $\mathrm{MSE}=0.3092$

Figure 7. Spline 0 circular image reconstruction with 128 uniformly distributed Katz angles on $\left[0, \pi\left[\right.\right.$ filtered by $k_{0}(b, p, q)$ and backprojected with a Mojette backprojection. The resulting images are normalized onto $[0,1]$

of both algorithms in situations where the high frequencies content are either constant (projections of the disk) or very different as for the square.

\section{CONCLUSION}

A new algorithm has been proposed. It is composed of a standard Conjugate Gradient algorithm expressed for our Mojette discrete geometry. The comparison with the FBP Reconstruction shows a good behavior of the CG reconstruction. The next step is to preconditionnate the Conjugate Gradient algorithm to converge faster. Our ultimate goal is to devise a 3D, fast iterative reconstruction technique for Mojette geometry data in the noisy data case.

\section{REFERENCES}

1. N. K. Bose and K. J. Boo. Asymptotic eigenvalue distribution of block-toeplitz matrices. IEEE Transactions on Information Theory, 44(2):858-861, March 1998.

2. Raymond H. Chan and Michael K. Ng. Conjugate gradient methods for Toeplitz systems. SIAM Review, 38(3):427-482, 1996.

3. Jeffrey A. Fessler and Scott D. Booth. Conjugate-gradient preconditioning methods for shift-invariant PET image reconstruction. IEEE Transaction on Image Processing, 8(5):688-699, May 1999.

4. JeanPierre Guédon and Yves Bizais. Bandlimited and haar filtered back-projection reconstuction. IEEE Transaction on Medical Imaging, 13(3):430-440, September 1994.

5. Jorge Nocedal and Stephen J. Wright. Numerical optimization. Springer Texts in Operations Research. Springer-Verlag, New York, NY, USA, 1999.

6. Myriam Servières, JeanPierre Guédon, and Nicolas Normand. A discrete tomography approach to PET reconstruction. In Yves Bizais, editor, Fully 3D Reconstruction In Radiology and Nuclear Medicine, June 2003.

7. Myriam Servières, Nicolas Normand, Yves Bizais, and JeanPierre Guédon. Noise behavior of spline mojette FBP reconstruction. In Proc. SPIE, Medical Imaging 2005: ImageProceding. SPIE, 2005. 